Themenheft Nr. 40: CoViD-19 und die digitale Hochschulbildung. Irritationen, Einsichten und Programmatiken

Herausgegeben von Markus Deimann, Marios Karapanos und Klaus Rummler

\title{
Zusammenhänge zwischen der Mediennutzung Studierender und ihrer Dozierenden an deutschen Hochschulen
}

\author{
Eine explorative Studie \\ Annalisa Biehl ${ }^{1}$ (D) und Kris-Stephen Besa ${ }^{1}$ (D) \\ ${ }^{1}$ Westfälische Wilhelms-Universität Münster
}

\begin{abstract}
Zusammenfassung
Die wachsende gesellschaftliche Bedeutung von Digitalisierung zeigt sich in den letzten Jahren auch durch die zunehmende Etablierung digitaler Strukturen innerhalb der deutschen Hochschulen. Dieser Prozess hat nicht zuletzt bedingt durch die Corona-Pandemie eine Beschleunigung erfahren. Abgesehen von infrastrukturellen Voraussetzungen kommt in diesem Kontext neben der Hochschulleitung gerade den Dozierenden durch die Implementierung digitaler Lehr- und Lernformate innerhalb von Lehrveranstaltungen eine zentrale Funktion hinsichtlich der Umsetzung und Ausgestaltung von Digitalisierung an Hochschulen zu. An diese Prämisse anknüpfend ist es Ziel des vorliegenden Beitrags, auf Basis einer Befragung 171 Studierender deren studienbezogene Mediennutzung zum Zeitpunkt vor der Pandemie zu erfassen. Darüber hinaus soll das lehrveranstaltungsbezogene Medienhandeln von Dozierenden, erfasst durch eine Fremdeinschätzung der Studierenden, untersucht werden, um anschliessend mittels Korrelationsanalysen auf mögliche Zusammenhänge zwischen der Nutzung digitaler Anwendungen beider Akteursgruppen zu schliessen. Dazu soll insbesondere der Einbezug forschungs- und literaturbezogener Anwendungen in den Blick genommen werden. Die Ergebnisse werden abschliessend vor dem Hintergrund der veränderten Lehrsituation durch die Corona-Pandemie diskutiert.
\end{abstract}

Correlations Between Media Use of Students and their Lecturers at German Universities. An Explorative Study

\begin{abstract}
The growing social importance of digitization is also reflected by the expansion of digital structures within German universities in recent years. This process has accelerated due to the CoViD-19-pandemic. Apart from infrastructural requirements, in this context it is not only the university management but also the lecturers who play a central role in the
\end{abstract}


implementation and design of digitization at universities by implementing digital teaching and learning formats within their classes. Following this premise, the aim of this paper is to examine student media use in the university context at the time before the pandemic on the basis of a survey of 171 students. In addition to the class-related media activities of lecturers' assessed by the students, possible correlations between the use of digital applications by both groups shall be analysed. For this purpose, the inclusion of researchand literature-related software applications will be considered in particular. Finally, the results will be discussed against the background of the changed teaching situation due to the CoViD-19-pandemic.

\section{Einleitung}

Digitale Medien haben sich in den letzten Jahren fest in den Lebensalltag integriert, was sich auch in der stetig steigenden Nutzungshäufigkeit dieser niederschlägt (vgl. Friedrichsen und Wersig 2020; Herzig und Martin 2018). Insbesondere junge Menschen verstehen digitale Medien als ständige Lebensbegleiter und Instrumente zur Lebensbewältigung, sodass sich diese nicht mehr aus dem Alltag wegdenken lassen. Angesichts der Corona-Pandemie beschleunigen sich gesellschaftliche Digitalisierungsprozesse und berühren dabei auch die Strukturen innerhalb der Hochschulen, wodurch sich neue, dauerhafte Aufgaben und Herausforderungen auf allen hochschulischen Ebenen ergeben. Neben den Hochschulleitungen kommt im Hinblick auf die konkrete Ausgestaltung digitaler Prozesse insbesondere den Lehrenden eine zentrale Funktion zu (vgl. KMK 2019; Persike und Friedrich 2016). Durch die Implementierung von digital gestützten Lehr- und Lernformaten haben diese einen möglicherweise nicht geringen Einfluss auf das Medienhandeln von Studierenden, wodurch sie eine entscheidende Multiplikatorenrolle einnehmen könnten. Vor diesem Hintergrund sollen neben der Etablierung digitaler Technologien in der Lehre auch mögliche Zusammenhänge zwischen dem Medienhandeln von Dozierenden und Studierenden untersucht und nachvollzogen werden. Besonders interessiert dabei die Untersuchung von Korrelationen zwischen der Nutzung von Forschungssoftware sowie Literaturdatenbanken seitens der Studierenden und ihrer Lehrenden. Es kann angenommen werden, dass mit dem vermehrten, durch einen potentiell kompetenten Einsatz der Dozierenden bedingten studentischen Gebrauch gerade letzterer Anwendungen eine gesteigerte Informationskompetenz der Studierenden einhergeht, die durchaus auch als Teilaspekt von Medienkompetenz verstanden werden kann, wenngleich darauf hingewiesen sei, dass von der ausschliesslichen Nutzung von forschungs- und literaturbezogenen Anwendungen nicht zwangsläufig auf einen kompetenten Umgang damit geschlossen werden kann. Hinweise auf eine potentielle Einflussnahme digitalgestützter Lehre auf das studentische Medieninteresse und -handeln würden somit die Bedeutung des Dozierendenhandelns dahingehend 
aufzeigen, als dass diese durch ihre Wirkung auf die Studierenden auch zu einem informations- und medienkompetenteren Umgang mit digitalen Formaten beitragen (können).

\section{Theoretischer Hintergrund}

Unter Digitalisierung versteht man die Verlagerung von Leistungen aus der analogen, physischen Welt in eine digitale, auf Informations- und Kommunikationstechnologie basierende Welt, mit der tiefgreifende gesellschaftliche Veränderungen einhergehen (von der Heyde et al. 2017; Wolf und Strohchen 2018). In Bezug auf die Digitalisierung der Hochschule lassen sich die damit einhergehenden Gestaltungsaufgaben in die Bereiche Forschung, Lehre/Lernen und Verwaltung differenzieren (vgl. Gilch et al. 2019; von der Heyde et al. 2017; KMK 2019). Hinsichtlich der Lehre wird neben der Etablierung von Learning-Management- und Online-Prüfungssystemen auf die curriculare (z. B. Umgang mit Veränderungsprozessen durch die Digitalisierung) sowie die didaktische (z. B. Einbezug digitaler Lehr-Lernformate) Verankerung von Digitalisierung gezielt (vgl. KMK 2019; von der Heyde et al. 2017).

Erprobungs- und Umsetzungsprozesse der Digitalisierung begannen Ende der 1990er Jahre, angetrieben durch erste staatliche Fördermassnahmen (Hofhues et al. 2020; Getto und Schulenburg 2018). Während in den ersten Jahren die Erforschung der Wirksamkeit der digitalen Lehre fokussiert wurde, zielen die staatlichen Förderprogramme nun zunehmend auf die strategische Hochschulentwicklung im Kontext der Digitalisierung (Getto und Kerres 2015). Analog dazu waren in den 2000er Jahren überwiegend noch keine Digitalisierungsziele in Strategieplänen etabliert (siehe z. B. Kleimann und Wannemacher 2004; Werner 2006), sondern hielten erst in den letzten Jahren unter anderem durch Forderungen von politischer (CDU/CSU/SPD 2018; KMK 2017; KMK 2019) sowie wissenschaftlicher Seite (z. B. durch den Wissenschaftsrat 2017) Einzug in die hochschulischen Entwicklungspläne oder wurden durch externe Konzeptpapiere festgehalten (vgl. Hochschulforum Digitalisierung 2016; Gilch et al. 2019). Vor allem aufgrund der Abstraktheit des Digitalisierungsbegriffs (Hofhues et al. 2020) wird die Festschreibung konkreter Ziele als wichtige Voraussetzung zur Ausgestaltung digitaler Infrastrukturen an den Hochschulen betrachtet (Pensel und Hofhues 2017; Schmid und Bäßler 2016; Hochschulforum Digitalisierung 2016). Eine umfassende Untersuchung von Gilch et al. (2019) zeigt, dass 54,5\% der befragten Hochschulen eine Digitalisierungsstrategie haben.

Während die tatsächliche Qualität der Digitalisierung in den Bereichen Lehre, Forschung und Verwaltung in der Untersuchung von Gilch et al. (2019) nur von 20\% der befragten Hochschulleitungen als hoch eingeschätzt wird, kommen Schmid et al. (2017) zu teilweise gegensätzlichen Ergebnissen. Die Befragten bewerten die Qualität der digitalen Infrastruktur überwiegend als gut bis sehr gut, jedoch sehen 
sie die didaktischen Potentiale der Digitalisierung in der Lehre oft ungenutzt. Darüber hinaus zeigen sich in anderen Untersuchungen hinsichtlich des Bereichs Lehren und Lernen noch starke inter- als auch intrahochschulische Disparitäten (Gilch et al. 2019; Persike und Friedrich 2016). Bei den Studierenden trifft man auf eine generelle Zufriedenheit mit der Qualität der Digitalisierung an Hochschulen (vgl. z. B. Persike und Friedrich 2016), was möglicherweise auch dazu führt, dass im Gegensatz zur schulischen Situation keine dauerhaften Probleme der Hochschulen hinsichtlich der digitalen Lehre während der Corona-Pandemie berichtet werden (Dreyer 2020). In Anlehnung an Hofhues, Pensel und Möller (2018) zeigt sich in Bezug auf hochschulinterne Digitalisierungsprozesse unter anderem die Herausforderung, dass sich die Zuständigkeiten innerhalb der Hochschule häufig als ungeklärt herausstellen, auch da die Zielvorgaben mitunter nicht in bzw. mit der Hochschule, sondern extern gesetzt werden (Hochschulforum Digitalisierung 2016). Hochschulleitungen kommt daher eine zentrale Steuerungsfunktion zu, die wegweisend für die konkrete Implementierung digitaler Lehrelemente seitens der Dozierenden ist (Hofhues, Pensel, und Möller 2018; Schmid et al. 2017; Wannemacher et al. 2016). Es gilt festzuhalten, dass digitale Strategien und Strukturen insbesondere in den letzten Jahren Einzug in die deutschen Hochschulen fanden. Jedoch folgt aus dem Vorhandensein digitaler Infrastrukturen keineswegs zwangsläufig eine Nutzung dieser seitens der Lehrenden (Brahm, Jenert, und Eulert 2016; Persike und Friedrich 2016; Getto und Kerres 2017; Gilch et al. 2019).

\subsection{Mediennutzung von Dozierenden in Lehrveranstaltungen}

Bereits in den frühen 2000er Jahren finden sich gezielte Versuche, Lehrende an Hochschulen für den Umgang mit digitalen Medien zu qualifizieren (z. B. Dusch und LütkeEntrup 2004). Neben einer möglichen Verbesserung der Lehrqualität soll durch die Mediennutzung von Hochschullehrenden darüber hinaus ein Beitrag für die Weiterentwicklung der Informationsgesellschaft geleistet werden (Dusch und Lütke-Entrup 2004; Eichhorn, Müller, und Tillmann 2017). Wenig überraschend ist, dass die Medienkompetenz der Hochschullehrenden als zentraler Faktor für einen didaktisch sinnvollen Einsatz digitaler Medien in der universitären Lehre gesehen wird (Aichinger 2018). Medienkompetenz umfasst dabei die souveräne Bedienung von und kritische Auseinandersetzung mit (digitalen) Medien (Hugger 2008; Süss, Lampert, und Trültzsch-Wijnen 2018), wobei das Konstrukt in der empirischen Forschung durchaus unterschiedlich operationalisiert wird und sich mitunter auch auf das Interesse für und Einstellungen gegenüber neue Medien bezieht (z. B. Biermann 2009; Kammerl und Mayrberger 2011; Palkowitsch-Kühl 2018). Eine gezielte Auseinandersetzung mit Medien in der universitären Ausbildung ist bislang allerdings kaum systematisch verankert, sodass Schwerpunkte und die explizite Beschäftigung mit Digitalisierung in 
erster Linie vom Individualinteresse der Lehrenden am jeweiligen Standort abhängen (Rott 2014). Die digitalen Kompetenzen von Hochschullehrenden sollen es ermöglichen, sowohl die Reflexion der Studierenden über die in der universitären Lehre eingesetzten Medien anzuregen als auch die eigene Anwendung aller Studierenden durch selbsttätige Aneignung zu befördern. Patentrezepte für die sinnvolle digitale Gestaltung gibt es jedoch nicht und die Spannbreite möglicher Szenarien reicht von der Nutzung von Lernplattformen, der Digitalisierung der Präsenzlehre oder digital gestütztem Austausch bis hin zu sog. MOOCs (Massive Open Online Courses) als nur einige der möglichen Realisierungen (Krauskopf und Zahn 2015). Auf weniger interessierte Lehrende können solche Ansprüche und Formate durchaus abschreckend wirken. Aber auch bei engagierten Lehrenden können die ständigen Neuerungen und stärker wachsenden Ansprüche zu Resignation führen (Wedekind 2008). Die Ausprägung der Nutzung von Möglichkeiten digitaler Hochschullehre scheint ebenfalls stark fächerabhängig und ist gerade in den im späteren Berufsfeld (derzeit) noch weniger digitalisierten Sozialwissenschaften geringer ausgeprägt (Krauskopf und Zahn 2015; Persike und Friedrich 2016).

Wie es um die tatsächliche Ausprägung der Medienkompetenz von Lehrenden an Universitäten bestellt ist, scheint bislang unklar und es liegen nur wenige hochschulspezifische Studien und Konzepte zur Medienkompetenz und -nutzung der dort Lehrenden vor. Mit Blick auf die Lehrpersonenausbildung konnten Capparozza und Irle (2020) lediglich 15 Arbeiten in ihr Review zur Bedeutung digitaler Kompetenzen bei Lehrpersonenausbildenden für das Lehren mit digitalen Medien und zur Vorbereitung der angehenden Lehrpersonen auf das Unterrichten mit digitalen Medien einfliessen lassen, die nicht nur aus dem hochschulischen Bereich stammen, sondern zum Teil auch die Rolle von Mentorinnen und Mentoren mit beleuchten. Hingegen konnten im gleichen Zeitraum mehrere hundert Arbeiten identifiziert werden, die auf die Kompetenzen der Lehramtsstudierenden fokussierten. Ebenfalls bemerkenswert ist mit Blick auf die Studienlage, dass sich keine Studie aus dem deutschsprachigen Raum für das Review finden liess (Capparozza und Irle 2020). Auch wegen der überschaubaren Studienlage orientieren sich konzeptionelle Arbeiten zur Medienkompetenz von Hochschullehrenden häufig an Überlegungen aus dem schulischen Kontext (z. B. Eichhorn, Müller, und Tillmann 2017). Legt man eine grundsätzliche Übertragbarkeit der Ergebnisse auf die universitäre Ebene zugrunde, so ist davon auszugehen, dass digitale Medien vor allem dann Verwendung in der Lehre finden, wenn die jeweiligen Lehrpersonen die Annahme einer positiven Unterstützung von Lernprozessen mit ihnen verbinden (Herzig und Martin 2018; Petko und Döbeli Honegger 2011) und sich kompetent im Umgang fühlen (Eickelmann, Lorenz, und Endberg 2016). Einschränkend muss zu dieser Überlegung jedoch ergänzt werden, dass ein stärkerer Einsatz digitaler Medien nicht zwangsläufig mit einer höheren Medienkompetenz gleichzusetzen ist. Ähnliche Erkenntnisse mit Blick auf Dozierende an Hochschulen zeigt die 
Befragung von Riedel und Börner (2018), die vor allem den didaktischen Nutzen und die selbsteingeschätzten Fähigkeiten als relevante Bedingungen für den Einsatz digitaler Medien von Lehrenden an deutschen Hochschulen identifizieren. Neben hohem Wissen über sind auch positive Einstellungen zu digitalen Medien eine Voraussetzung, um diese in ihre Lehre mit einfliessen zu lassen. Dabei wird es möglich, positive Einflüsse auf die Fähigkeiten im Umgang mit digitalen Medien der Studierenden nachzuweisen. Diese können sowohl durch die Kompetenzen von Lehrpersonen als auch durch direkte Interventionen, beispielsweise auf Basis angepasster, interaktiver digitaler Lehrmaterialien zur Nutzung in kurzzeitigen Fördersegmenten zur Medienkompetenz in hochschulischen Settings, beeinflusst werden (Bergstrom, Flynn, und Craig 2018; Capparozza und Idel 2020).

\subsection{Mediennutzung von Studierenden}

Der studienbezogenen Nutzung digitaler Anwendungen ist der studentische Besitz digitaler Endgeräte vorausgesetzt. Grosch und Gidion stellen bereits 2011 eine Ablösung des stationären PCs durch den Laptop fest, der sich in den folgenden Jahren als das von den Studierenden am häufigsten genutzte digitale Endgerät etabliert (vgl. Schäffer 2015). Während schon 2012 ca. 90\% der Studierenden im Besitz eines Laptops waren, beschränkt sich der eines Smartphones auf circa die Hälfte der Studierenden (vgl. Schäffer 2015; Zawacki-Richter, Cramer, und Müskens 2016). In den folgenden Jahren etablierte sich das Smartphone zunehmend, sodass 2015 gleich viele (vgl. Zawacki-Richter et al. 2016), im Jahr 2018 bereits mehr Studierende ein Smartphone $(96,4 \%)$ als einen Laptop $(93,5 \%)$ besassen (vgl. Pumptow und Brahm 2020). Die Nutzendenzahlen von Tablets entwickelten sich in etwa proportional zu denen der Smartphones (vgl. Schäffer 2015; Zawacki-Richter et al. 2016), wenngleich erstere 2018 nur von knapp der Hälfte der Studierenden in Anspruch genommen wurden (vgl. Pumptow und Brahm 2020). Hinsichtlich der studienbezogenen Nutzungsintensität zeigt sich im zeitlichen Verlauf ebenso eine Zunahme (vgl. Zawacki-Richter et al. 2016), deren Ursache in der flächendeckenden Etablierung digitaler Endgeräte und Anwendungen im privaten Besitz der Studierenden als auch innerhalb der Hochschule zu vermuten ist. Die Mehrheit der Studierenden lässt sich als «Unterhaltungsnutzer» typologisieren, die gegenüber den «Zwecknutzern» weniger E-Learning-Tools oder Software, sondern eher studienbezogene Chat-Dienste und Soziale Netzwerke nutzen (vgl. Vogelsang et al. 2019; Zawacki-Richter et al. 2016). Begründet könnte dies mit der zunehmenden Etablierung des Smartphones werden. Ferner wird trotz guter digitaler Ausstattung der Studierenden, Angebotsbereitstellung durch die Hochschule und privater Nutzungsvielfalt zum studienbezogenen Lernen überwiegend auf digitale Standardprogramme zurückgegriffen (vgl. Grosch 2012; Persike und Friedrich 2016; Steffens, Schmitt, und Aßmann 2017; Zawacki-Richter 
et al. 2016). Explizit für Lehr- und Lernzwecke entwickelte Tools wie z. B. Lern-Apps werden von den meisten Studierenden überwiegend nie benutzt (vgl. Steffens et al. 2017). Im Hinblick auf die Informationskompetenz von Studierenden zeigen sich ähnliche Befunde. Jene umfasst die studentische Kompetenz, einen konkreten Informationsbedarf formulieren, sich effizient Zugang zu den benötigten Informationen verschaffen als auch diese bewerten und kontextualisieren zu können (Deutscher Bibliotheksverband e.V. 2009). Fachzeitschriften werden dabei nur selten (Gidion und Weyrich 2017), Suchmaschinen wie z. B. Google sowie Plattformen wie Wikipedia jedoch häufig zur Recherche herangezogen (vgl. Grosch und Gidion 2011; Günther 2016; Schmid et al. 2017; Steffens et al. 2017; Zawacki-Richter et al. 2016). Hinsichtlich der Nutzung von Literaturverwaltungsanwendungen gaben in einer Befragung von Günther (2016) mit circa 1200 Studierenden der TU Dresden 28\% an, diese schon einmal für das Studium genutzt zu haben, wenngleich 37\% der Aussage zustimmten, keine Literaturverwaltungsanwendungen zu kennen, und die Nutzendenzahlen gegenüber anderen Anwendungen verhältnismässig gering ausfielen. Insgesamt geschieht die Nutzung digitaler Medien wenig zielgerichtet zu Lernzwecken (Vogelsang et al. 2019), sodass - so fassen Zawacki-Richter et al. (2016) zusammen - der Begriff digital native «ohne jeden Beschreibungswert für den Hochschulbereich» (ebd., 37) sei. Zur Erklärung der geringfügig variablen und informationskompetenten Nutzung digitaler Anwendungen lässt sich das von Davis 1985 entwickelte Technology Acceptance Model (TAM) hinzuziehen (vgl. Davis 1985; Davis, Bagozzi, und Warshaw 1989). Es geht davon aus, dass die von einer Person wahrgenommene Benutzerfreundlichkeit (percieved ease of use) sowie der Nutzen (percieved use) massgebend für die Nutzungsabsicht und folglich die Nutzung einer Technologie sind. Diese Zusammenhänge wurden mehrfach empirisch belegt (z. B. Davis et al. 1989; Özbek et al. 2014; Taylor und Todd 1995). Analog zum TAM zeigen Zawacki-Richter et al. (2016), dass den befragten Studierenden vor allem die Zugänglichkeit (percieved ease of use) sowie die Nützlichkeit (percieved use) digitaler Medien und Anwendungen wichtig ist. Folglich könnte ein verstärkter Einbezug studentisch eher fremder Tools (z. B. Literaturverwaltungsanwendungen) in Lehrveranstaltungen möglicherweise zu einer Konventionalisierung wissenschaftsbezogener Anwendungen einerseits und zu einer Ablösung informeller studentischer Informationsquellen andererseits beitragen (vgl. Riplinger und Schiefer-Rohs 2017).

\subsection{Zusammenhänge zwischen der Mediennutzung von Studierenden und ihren Dozierenden}

Hinsichtlich der Untersuchung des Zusammenhangs zwischen dem Einbezug digitaler Medien in Lehrveranstaltungen und der studentischen Mediennutzung existieren bislang eher konzeptionelle Überlegungen als empirisch-quantifizierende Belege. 
Ausgehend von dem von Fend (1980) entwickelten und von Helmke und Weinert (1997) adaptierten Angebot-Nutzungs-Modell ist eine gänzliche Unabhängigkeit zwischen der Mediennutzung von Lehrenden und Lernenden unwahrscheinlich. Dozierende schaffen ein (mediales) Angebot, das von den Studierenden für Lernzwecke und Leistungserbringung genutzt werden kann, mitunter sogar muss. Ein Beleg findet sich in den Ergebnissen von Schmid et al. (2017), die älteren Studierenden eine signifikant höhere Motivation gegenüber der studienbezogenen Nutzung digitaler Technologien nachweisen, was auf den Einbezug derer in Lehrveranstaltungen zurückgeführt werden könnte. Es gilt jedoch darauf hinzuweisen, dass damit keine höhere Nutzungsintensität einzelner Technologien verbunden ist (ebd.). Zudem existieren ebenso Arbeiten, die keine Alterseffekte feststellen (vgl. Grosch und Gidion 2011; Persike und Friedrich 2016). Einen empirischen Hinweis auf den Zusammenhang zwischen der Mediennutzung von Dozierenden und Studierenden geben Vogelsang et al. (2019), die den Lernerfahrungen von Lehramtsstudierenden aus universitären Veranstaltungen einen positiven Zusammenhang mit den Einstellungen und Selbstwirksamkeitserwartungen zum eigenen Medieneinsatz nachwiesen. Interessant ist ferner, dass in der Untersuchung von Gidion und Weyrich (2017) 72\% der Studierenden angaben, nie Lern-Apps zu benutzen, während Schmid et al. (2017) gleichzeitig eine geringfügige Nutzung dieser (3\%) durch Hochschullehrende in Lehrveranstaltungen feststellten. Auf einen Zusammenhang deuten ebenso die ähnlich hohen WikipediaNutzungsquoten von Dozierenden und Studierenden hin (vgl. Schmid et al. 2017). Gleichzeitig berichten Kommer und Biermann (2012), dass die in ihrer Studie befragten Lehramtsstudierenden das Gefühl haben, medienpädagogische Kenntnisse und Fähigkeiten im Laufe der universitären Ausbildung nur unzureichend zu erwerben, obwohl die Mehrheit medienpädagogischen Themen eine hohe Relevanz zuschreibt. Goode (2010) vermutet, dass das Mediennutzungsverhalten von Studierenden primär durch ausseruniversitäre Einflüsse bereits vor dem Universitätseintritt festgelegt wird, sodass bei Studienbeginn bereits eine «technology identity» bestünde, die sich im Studienverlauf sogar noch weiter festschreiben würde. Es lässt sich resümieren, dass angesichts der skizzierten Hinweise ein Zusammenhang zwischen der Mediennutzung Studierender und ihrer Dozierenden vermutet werden kann, der jedoch noch nicht umfangreich empirisch und technologiespezifisch differenziert untersucht wurde. An dieser Stelle setzt die folgende Untersuchung an.

\section{Untersuchungshypothesen}

Anknüpfend an das oben skizzierte Forschungsdesiderat soll in dem vorliegenden Beitrag der Frage nachgegangen werden, inwieweit digitalgestützte Lehre an deutschen Hochschulen etabliert ist. Dazu soll neben dem generellen studienbezogenen Mediennutzungsverhalten von Dozierenden sowie von Studierenden untersucht 
werden, inwiefern bereichsspezifische, insbesondere literatur- und forschungsbezogene Software in der Lehre von Bedeutung ist. Darüber hinaus soll das studienbezogene Mediennutzungsverhalten von Studierenden hinsichtlich möglicher Zusammenhänge mit der lehrveranstaltungsbezogenen Mediennutzung seitens der Dozierenden überprüft werden.

Es wird dabei angenommen, dass

- trotz der grösstenteils vorhandenen digitalen Infrastruktur die Nutzung digitaler Lehrformate eher gering ausgeprägt ist.

- die Mediennutzung Hochschullehrender mit der ihrer Studierenden zusammenhängt.

\section{Studiendesign, Stichprobe und methodisches Vorgehen}

Zur Beantwortung der Forschungsfragen wurde auf die Daten einer Online-Befragung zurückgegriffen, die an mehreren Universitäten in Deutschland (Hildesheim, Trier, Münster, Dortmund) in Master-Seminaren verschiedener Fächer beworben wurde, um ein möglichst breites Abbild der Studiensituation zu Beginn des Semesters zu bekommen, das nicht auf etwaige Standortspezifika zurückzuführen ist. Insgesamt konnten so Angaben von 171 Masterstudierenden erhoben werden, die über zahlreiche Studienfächer und Fachrichtungen (u. a. naturwissenschaftliche Fächer, Psychologie, Sprachen) streuen, wobei ein hoher Anteil Lehramtsstudierender $(55,6 \%)$ in der Stichprobe vorhanden ist und - ggf. auch damit zusammenhängend - ein etwas erhöhter Anteil weiblicher Studienteilnehmender (62,6\%). Das Medianalter lag bei 24 Jahren, der Median der Abiturnote bei 2,2 (gut). Die Befragung fand zu Beginn des Sommersemesters 2020 in den ersten vier Semesterwochen statt und somit im ersten «Digitalsemester». Da jedoch, um einen möglichen Längsschnitt zu realisieren und Verzerrungen durch den Messzeitpunkt zu verringern, die vorher bestehende Situation erfasst werden sollte, wurden die Studierenden bei der Beantwortung explizit dazu aufgefordert, ihre Einschätzungen auf die Zeit vor dem Sommersemester 2020 und damit vor die gezwungenermassen veränderte Lehrsituation während der CoViD-19-Pandemie zu beziehen (zur möglichen Problematik retrospektiver Befragung siehe Dürnberger, Drasch, und Matthes 2011).

Zur Erfassung des Medieninteresses wurde eine etablierte Skala aus den ICILSStudien genutzt (Skala zur Erfassung von Interesse und Vergnügen am Umgang mit IT: Gerick et al. 2018). Die 11 Items umfassende, eindimensionale Skala zeigt mit einem $\alpha=.91$ eine sehr gute interne Konsistenz (Beispielitem: «Ich benutze Computer, weil mich die Technik sehr interessiert.»). Für die Operationalisierung des eigenen Medienhandelns der Studierenden und des durch die Studierenden fremdeingeschätzten Medienhandelns der Universitätsdozierenden wurde auf Eigenentwicklungen zurückgegriffen. Erfasst wurden von den Studierenden die generelle Nutzung von 
digitalen Medien im universitären Lern- und Arbeitskontext (3 Items, $\alpha=.72$; Bsp.: «Individuelle Erstellung eigener Medienprodukte, z. B. Powerpoint für Vortrag») sowie die Nutzung von forschungsbezogener Software und Literaturdatenbanken (4 Items, $a=.67$; Bsp.: «Individuelle Nutzung von Literaturverwaltungssoftware, z. B. Citavi»). Daneben interessierte als Äquivalent zu den Studierendenskalen der wahrgenommene Medieneinsatz von den Dozierenden, für den eine Skala zur Fremdeinschätzung der Nutzung von Literaturdatenbanken und Forschungssoftware eingesetzt wurde (4 Items, $\alpha=.70$; Bsp.: «Nutzung von Literaturverwaltungssoftware für das Seminar, z. B. geteilte Citavi-Datenbanken»), sowie zur generellen Nutzung digitaler Medien und Plattformen in der Lehre (9 Items, $\alpha=.64$; Bsp.: «Nutzung von Videochats via Zoom, Skype etc.»). Die Skala zur Mediennutzung der Hochschullehrenden fällt etwas umfangreicher aus als die Studierendenskala, da dort unter anderem auch die Nutzung digitaler Prüfungsformate miterfasst wurde, für die auf Studierendenseite keine Entsprechung vorhanden ist. Die internen Konsistenzen der eigens konstruierten Skalen wurden als gerade noch zufriedenstellend eingeschätzt. Sämtliche Beantwortungen der Selbst- und Fremdeinschätzung erfolgten auf 5er-Likert-Antwortskalen mit den Polen $1=$ «stimme gar nicht zu» bis $5=$ «stimme völlig zu» für das Medieninteresse bzw. den Polen 1 = «gar nicht» bis 5 = «fast immer» für die Skalen zur Nutzungshäufigkeit von Medien. Darüber hinaus wurden die Studierenden nach der Kenntnis und dem Einsatz weiterer spezifischer digitaler Anwendungen in der Lehre vor dem Sommersemester 2020 befragt. Diese konnten frei benannt werden und sollten ebenfalls hinsichtlich der Nutzungshäufigkeit auf einer 5er-Skala eingeschätzt werden.

\section{Ergebnisse}

Die Mittelwerte zeigen, dass lediglich geringe Ausprägungen bei den erhobenen Skalen zur Mediennutzung vorliegen. Sowohl die eigene Mediennutzung der Studierenden als auch die Nutzung digitaler Medien durch die Dozierenden aus Studierendensicht liegen unter dem theoretischen Skalenmittel von 3. Dies signalisiert eine tendenziell seltene Nutzung digitaler Anwendungen. Solches gilt insbesondere für die Nutzung von Literaturdatenbanken und forschungsbezogener Software, die noch geringere Werte aufweisen als die Nutzung von Medien durch die Studierenden bzw. die weitere Nutzung digitaler Medien von Hochschuldozierenden. Das Medieninteresse hingegen ist deutlich stärker bei den Studierenden ausgeprägt und liegt über dem theoretischen Skalenmittel. 


\begin{tabular}{|l|c|}
\hline Skala & Mittelwert (SD) \\
\hline Medieninteresse & $3,24(0,08)$ \\
\hline generelle Mediennutzung Studierende & $1,64(0,75)$ \\
\hline Literaturdatenbanken und Forschungssoftware Studierende & $1,53(0,65)$ \\
\hline generelle Mediennutzung Dozierende & $2,16(0,57)$ \\
\hline Literaturdatenbanken und Forschungssoftware Dozierende & $1,86(0,84)$ \\
\hline
\end{tabular}

Tab. 1.: Mittelwerte der Skalen zu Medieninteresse und Mediennutzung.

Dieser eher gering einzuschätzende Medieneinsatz bestätigte sich auch mit Blick auf die Frage nach dem wahrgenommenen Einsatz verschiedener digitaler Tools für die Online-Lehre. Von den 171 befragten Studierenden gab knapp jede bzw. jeder Fünfte an, über die in den Skalen genannten Anwendungen hinaus noch weitere digitale Lehrformate zu kennen. Von den Studierenden noch am häufigsten genannt wurden Programme mit Rückmeldungs- und Quizfunktion wie z. B. die Internetplattformen Kahoot! ( $n=8)$ oder Mentimeter $(n=8)$, universitäre Lernplattformen wie Moodle oder Learnweb $(n=7)$ sowie Software für Videokonferenzen, insbesondere Zoom Video Communications $(n=7)$. Noch weniger etabliert sind Flipped-ClassroomAnwendungen $(n=3)$, kollaborative Tools wie Microsoft Teams $(n=2)$ oder Slack ( $n$ $=1)$, Gamification-Software $(n=2)$ sowie fachspezifische Programme wie Geogebra $(n=2)$ oder MATLAB $(n=1)$. Auffällig ist ferner, dass von den Studierenden, die angaben, weitere als die in den Skalen genannten Anwendungen zu kennen, überwiegend mehr als ein digitales Tool benennen konnten. Hinsichtlich der Nutzungshäufigkeit der verschiedenen Anwendungen dominieren analog zum Bekanntheitsgrad didaktik- bzw. lehrveranstaltungsbezogene Programme wie Kahoot $(\bar{x}=3,6)$, Mentimeter $(\bar{x}=3,0)$ oder Zoom $(\bar{x}=3,6)$. Speziellere Tools sind nicht nur unbekannter, sondern werden auch weniger häufig genutzt, z. B. Flipped-Classroom-Anwendungen $(\bar{x}=1,7)$ oder Gamification-Software $(\bar{x}=1,5)$.

Die durchgeführte bivariate Korrelationsanalyse zeigt jedoch trotz der insgesamt geringen Ausprägung deutliche Zusammenhänge zwischen dem fremdeingeschätzten Medieneinsatz der Dozierenden und der jeweiligen Nutzung von Studierenden.

\begin{tabular}{|l|c|c|c|c|c|}
\hline & MI & gNS & LFS & gND & LFD \\
\hline MI & & & & & \\
\hline gNS & .09 & & & & \\
\hline LFS & .09 & $.32^{\star \star}$ & & & \\
\hline gND & .06 & $.39^{\star \star}$ & $.25^{\star \star}$ & & \\
\hline LFD & .18 & $.33^{\star \star}$ & $.62^{\star \star}$ & $.45^{\star \star}$ & \\
\hline
\end{tabular}

Tab. 2.: Korrelation der Skalen zu Medieninteresse und Mediennutzung. Anmerkung: $\mathrm{MI}=\mathrm{Me}-$ dieninteresse, gNS = generelle Mediennutzung Studierende, LFS = Literaturdatenbanken und Forschungssoftware Studierende, gND = generelle Mediennutzung Dozierende, $\mathrm{LFD}=$ Literaturdatenbanken und Forschungssoftware Dozierende; ${ }^{*}=p<.05,{ }^{* \star}=p<.01$. 
Es sind dabei vor allem die «benachbarten» Konstrukte zwischen den Selbst- und Fremdeinschätzungen, die enger miteinander zusammenhängen. So zeigt sich der höchste Korrelationskoeffizient für den Zusammenhang zwischen der fremdeingeschätzten Nutzung von Literaturverwaltungs- und Forschungssoftware durch die Dozierenden und der entsprechenden Studierenden-Selbsteinschätzung hinsichtlich der Nutzung. Hier liegt der Korrelationskoeffizient über einem Wert von .5, was einen starken linearen Zusammenhang in den Konventionen Cohens (1988) darstellt. Bei den übrigen signifikanten Koeffizienten liegen Zusammenhänge im mittleren Bereich vor. Bei der generellen Nutzung von digitalen Medien besteht äquivalent hierzu ebenfalls der höchste positive Zusammenhang zwischen den Fremdeinschätzungen des Dozierendenhandelns und der eigenen Aktivität der Mediennutzung im Rahmen universitärer Lehre. Das Medieninteresse spielt hierfür jedoch keine relevante Rolle.

\section{Diskussion}

Die vorliegenden Ergebnisse zeigen, dass zum Zeitpunkt der Erhebung bzw. für den durch die Befragung anvisierten Zeitraum von digital-gestützter Hochschullehre nur in sehr geringem Ausmass die Rede sein kann. Trotz hohen studentischen Medieninteresses fällt die studiumsbezogene Nutzung von digitalen Medien seitens der Studierenden, wie auch die fremdeingeschätzte, lehrveranstaltungsbezogene Nutzung digitaler Anwendungen durch Dozierende gering aus. Studierende können nur wenige spezifische digitale Formate benennen, die gleichzeitig in den Lehrveranstaltungen nur geringfügige Verwendung finden. Insbesondere Literaturdatenbanken und Forschungssoftware scheinen selten etablierte Gegenstände in der Hochschullehre zu sein. Im weiteren zeigen sich sowohl für die übergreifende wie auch für die forschungs- und literaturverwaltungsbezogene Mediennutzung Zusammenhänge zwischen den Studierenden und ihrer Dozierenden, während das studentische Medieninteresse mit diesen Konstrukten nicht korreliert.

Das im Verhältnis zur studienbezogenen Mediennutzung hohe Interesse an digitalen Medien seitens der Studierenden könnte auf die in empirischen Arbeiten nachgewiesene Etablierung und Nutzung von digitalen Medien im Privaten (vgl. z. B. Zawacki-Richter et al. 2016) zurückzuführen sein. Die befragten Studierenden sind digitalen Medien zwar zugewandt, jedoch schlägt sich dies nicht in der lernbezogenen Nutzung insbesondere spezieller Anwendungen (wie literatur- und forschungsbezogener Software) nieder, was sich auch unter Einbezug des Technology Acceptance Model (vgl. Davis 1985; Davis, Bagozzi, und Warshaw 1989) erklären lässt. Im Hinblick auf literatur- und forschungsbezogene Anwendungen ist davon auszugehen, dass die Bedienung schwieriger und der wahrgenommene Nutzen von den Studierenden eher gering eingeschätzt werden, da deren Gebrauch im (Studien-)Alltag möglicherweise überwiegend fakultativ und nicht obligatorisch-notwendig ist. Damit liesse 
sich auch erklären, dass die bekanntesten und überwiegend am häufigsten verwendeten digitalen Anwendungen sich auf lehrveranstaltungs- bzw. didaktikbezogene Programme (z. B. Kahoot!, Mentimeter oder Zoom Video Communications) und universitäre Lernplattformen beschränken, die für das Studium von täglichem, essentiellem Nutzen sind. Programme wie Microsoft Teams oder Slack, die weniger direkt auf die Unterstützung der Didaktik zielen, sondern eher ausserhalb von Lehrveranstaltungen relevant werden, sind bei den befragten Studierenden weniger bekannt (vgl. auch Steffens et al. 2017) und werden im Kontext von Lehrveranstaltungen auch weniger häufig genutzt. Analog dazu wird in empirischen Arbeiten zu den Dozierenden der didaktische Nutzen und die Relevanz der Unterstützung von Lernprozessen hinsichtlich des Einbezugs digitaler Medien hervorgehoben (vgl. Herzig und Martin 2018; Petko und Döbeli Henegger 2011; Riedel und Börner 2018). Letzteres könnte ebenso die hier nachgewiesene aus studentischer Sicht geringe Nutzung literaturund forschungsbezogener digitaler Anwendungen seitens der Dozierenden erklären. Diese verorten den Nutzen digitaler Elemente möglicherweise eher in der Didaktik, wodurch in der Lehre weniger auf spezielle forschungs- und literaturbezogene Programme zurückgegriffen wird. Insgesamt zeigt sich für die Zeit vor dem Digitalsemester weder auf Dozierenden- noch auf Studierendenseite ein stark etablierter Einsatz mediengestützter Lehr- und Lernformate.

Die Zusammenhänge zwischen den selbst- und fremdeingeschätzten Konstrukten sind insofern interessant, als dass die Nähe der Konstrukte Hinweise auf mögliche - in dieser Studie so nicht erfasste - Kausalzusammenhänge gibt. Zwar könnten auch digital affine Studierende vor allem in die Veranstaltungen entsprechend in ihrer Lehre aufgestellter Dozierenden streben, es scheint jedoch möglicherweise naheliegender, dass die Mediennutzung von Hochschuldozierenden die der Studierenden beeinflusst. Dieses gilt insbesondere für die Verwendung von Literaturverwaltungssoftware bzw. Software aus dem Bereich der Forschungsmethodik. Im Hinblick auf das Angebot-Nutzungs-Modell (vgl. Fend 1980; Helmke und Weinert 1997) ist es dabei allerdings möglich, dass sich dieser Einfluss gegebenenfalls gezwungenermassen ergibt, wenn ein spezifischer Medieneinsatz über die Leistungserbringung in Lehrveranstaltungen obligatorisch wird. Dieses erklärt dann allerdings nicht die Zusammenhänge zwischen der weiteren Mediennutzung von Dozierenden und Studierenden: Wobei auch hier eine Möglichkeit der Erklärung in studiengangsspezifischen Effekten besteht, wenn z. B. im naturwissenschaftlichen Bereich ein höherer Medieneinsatz von Dozierenden und Studierenden gefordert wird (vgl. z. B. Persike und Friedrich 2016). Um diesen Fragen vertiefend nachzugehen, bedarf es allerdings einer umfangreicheren Studierendengruppe. Die für diesen Beitrag genutzte Stichprobe zeichnet sich sowohl durch eine hohe Fächerheterogenität als auch eine unproportionale Verteilung hinsichtlich einzelner Studienfachrichtungen sowie dem starken Anteil an Lehramtsstudierenden aus. Mit Blick auf das Untersuchungsdesign ist anzumerken, 
dass es sich bei der Erhebung des Medieneinsatzes der Dozierenden um die Fremdeinschätzung der Studierenden handelt, wodurch eine Verzerrung der eigentlichen Nutzung durch die zwangsläufig subjektive Wahrnehmung der Studierenden nicht ausgeschlossen werden kann. Ebenso lässt sich hinterfragen, inwiefern die retrospektiv erhobenen Einschätzungen der Studierenden zur Mediennutzung valide den Zeitpunkt vor der erzwungenen Umstellung auf digitale Lehr- und Lernformate im Zuge der Corona-Pandemie widerspiegeln. Insbesondere hinsichtlich der Frage nach der Kenntnis weiterer digitaler Anwendungen könnten potenzielle Verzerrungen vermutet werden, obgleich der Befund, dass von den Studierenden nur wenige weitere digitale Anwendungen angegeben wurden, eher für das Gegenteil spricht. Darüber hinaus sei darauf verwiesen, dass die in der Untersuchung nachgewiesene, als durchaus gering zu bewertende Mediennutzung nicht mit einer defizitären Medienkompetenz sowohl von Studierenden als auch von Dozierenden gleichgesetzt werden kann. Zwar kann einerseits ein Kompetenzzuwachs durch die vermehrte Nutzung insbesondere forschungs- und literaturverwaltungsbezogener Anwendungen auch im Hinblick auf die Informationskompetenz Studierender vermutet werden, andererseits lässt sich zum Beispiel mit Blick auf die zwangsweise deutlich vermehrte Nutzung digitaler Lehr-und Lernformate durch die Corona-Pandemie kein tatsächlicher Kompetenzerwerb garantieren. Weitere Befragungen sollten die skizzierten Probleme unter anderem durch den Einbezug längsschnittlicher Designs stärker berücksichtigen. Eine Ausweitung der Befragung wurde zu Beginn des Sommersemesters 2021 vorgenommen und an ein quasi-experimentelles Design geknüpft, in dem der Aufbau digitaler Kompetenzen und die Entwicklung des Medieninteresses unter kontrollierter Berücksichtigung des Dozierendenhandelns mit Blick auf Softwarenutzung und Medieninhalte in Lehrveranstaltungen vorgenommen wird.

Vor dem Hintergrund der veränderten Lehrsituation durch die Corona-Pandemie wird zu beobachten sein, welche Veränderungen sich unter anderem in der Folgeerhebung zeigen. Neben einer deutlichen Zunahme an digitalen Lehrformaten und entsprechend auch vermehrten Arbeitsaufträgen und Anforderungen zur Nutzung von digitalen Tools in Lehre und studiumsbezogener Arbeit wird vor allem zu verfolgen sein, ob sich hierdurch auch ein gesteigertes Medieninteresse bei Studierenden einstellt. Darüber hinaus dürfte langfristig ebenfalls relevant sein, inwiefern die beobachteten Effekte über die Pandemie-Situation hinaus Bestand haben. Eine Rückkehr zum in dieser Studie skizzierten Status quo einer fast kaum vorhandenen Nutzung digitaler Elemente in Studium und Lehre ist dabei weder zu erwarten noch wünschenswert. Hier sind vor allem die Lehrenden an Hochschulen gefragt, den Anforderungen auch durch einen zeitgemässen Medieneinsatz Rechnung zu tragen. Es ist zumindest davon auszugehen, dass durch die neuartige Situation des Distanzlernens (und ggf. -prüfens) auf Seiten des hochschulischen Lehrpersonals nicht nur neue Techniken kennengelernt und beherrscht werden, sondern dass hierüber auch ein 
Zuwachs des Zutrauens in die eigenen Fähigkeiten im Umgang mit digitalen Medien und virtuellen, (a-)synchronen Lehrveranstaltungen hergestellt wird. Sowohl Vor- als auch Nachteile verschiedener Lehrelemente können dadurch kompetenter als zuvor gegeneinander abgewogen und entsprechend situationsspezifisch angemessen angewendet werden. Gleiches gilt an der Stelle auch für die Studierenden. Durch die vermehrte Lehre im digitalen Format sollten sich auch bei ihnen ähnliche Zuwächse im Kompetenzaufbau finden lassen - insbesondere bei variablem Vorgehen auf der 〈Angebotsseite〉-, wie dieses für die Lehrenden zu erwarten ist.

\section{Literatur}

Aichinger, Susanne. 2018. «Ausgewählte digitalisierte Elemente in der Hochschullehre». Haushalt in Bildung \& Forschung 7 (4): 33-43. https://doi.org/10.3224/hibifo.v7i4.03.

Bergstrom, Andrea M., Mark Flynn, und Clay Craig. 2018. "Deconstructing Media in the College Classroom: A Longitudinal Critival Media Literacy Intervention». The National Association for Media Literacy Education's Journal of Media Literacy Education 10 (3): 113 -131. https:// doi.org/10.23860/JMLE-2018-10-3-7.

Biermann, Ralf. 2009. Der mediale Habitus von Lehramtsstudierenden. Eine quantitative Studie zum Medienhandeln angehender Lehrpersonen. Wiesbaden: VS Verlag für Sozialwissenschaften. https://doi.org/10.1007/978-3-531-91501-2.

Brahm, Taiga, Tobias Jenert, und Dieter Eulert. 2016. «Pädagogische Hochschulentwicklung als Motor für die Qualitätsentwicklung von Studium und Lehre». In Pädagogische Hochschulentwicklung: Von der Programmatik zur Implementierung, herausgegeben von Taiga Brahm, Tobias Jenert und Dieter Eulert, 19-36. Wiesbaden: Springer VS. https://doi. org/10.1007/978-3-658-12067-2_2.

Capparozza, Marcel, und Gabriele Irle. 2020. «Lehrerausbildende als Akteure für die Digitalisierung in der Lehrerbildung. Ein Review». In Bildung im digitalen Wandel. Die Bedeutung für das pädagogische Personal und für die Aus- und Fortbildung, herausgegeben von Annika Wilmers, Carolin Anda, Carolin Keller und Marc Rittberger, 103-127. Münster: Waxmann. https://doi.org/10.31244/9783830991991.04.

CDU/CSU/SPD. 2018. «Ein neuer Aufbruch für Europa. Eine neue Dynamik für Deutschland. Ein neuer Zusammenhalt für unser Land. Koalitionsvertrag zwischen CDU, CSU und SPD. 19. Legislaturperiode». Berlin.

Cohen, Jacob. 1988. Statistical Power Analysis for the Behavioral Sciences. Hillsdale: Erlbaum.

Davis, Fred D. 1985. A Technology Acceptance Model for Empirically Testing New Information Systems. Massachusetts: Massachusetts Institute of Technology.

Davis, Fred D., Richard Bagozzi, und Paul R. Warshaw. 1989. «User Acceptance of Computer Technology: A Comparison of two Theoretical Models». Management Science 35 (8), 9821003. https://doi.org/10.1287/mnsc.35.8.982. 
Deutscher Bibliotheksverband e.V. 2009. «Standard der Informationskompetenz für Studierende». dbv Sektion IV, Frühjahrstagung, 25.-26.3.2009. https://www.bibliotheksverband.de/ fileadmin/user_upload/Kommissionen/Kom_Dienstleistung/Publikationen/Standards_Infokompetenz_03.07.2009_endg.pdf.

Dreyer, Malte. 2020. «Kurzumfrage zur Situation der Digitalen Angebote für Lehre und HomeOffice im April 2020 durch den ZKI-Arbeitskreis \& Organisation und die AMH». Berlin: Humboldt-Universität zu Berlin. https://doi.org/10.5281/zenodo.3826557.

Dusch, Christiane, und Monika Lütke-Entrup. 2004. «Die Qualifizierungsinitiative e-teaching@ university: Maßgeschneiderte Medienberatung für Lehrende an Hochschulen». In Medienkompetenz für die Hochschullehre, herausgegeben von Katja Bett, Joachim Wedekind und Peter Zentel, 93-109. Münster: Waxmann. https://www.e-teaching.org/news/termine/tagungen/berichte/dusch-le.pdf.

Dürnberger, Andrea, Katrin Drasch, und Britta Matthes. 2011. "Kontextgestützte Abfrage in Retrospektiverhebungen. Ein kognitiver Pretest zu Erinnerungsprozessen bei Weiterbildungsereignissen». Methoden, Daten, Analysen 5 (1): 3-35. https://nbn-resolving.org/ urn:nbn:de:0168-ssoar-255001.

Eichhorn, Michael, Ralph Müller, und Alexander Tillmann. 2017. «Entwicklung eines Kompetenzrasters zur Erfassung der „Digitalen Kompetenz“ von Hochschullehrenden.» In Bildungsräume. Proceedings der 25. Jahrestagung der Gesellschaft für Medien in der Wissenschaft, 5. bis 8. September 2017 in Chemnitz, herausgegeben von Christoph Igel, 209-219. Münster: Waxmann. https://doi.org/10.25656/01:16147.

Eickelmann, Birgit, Ramona Lorenz, und Manuela Endberg. 2016. «Die Relevanz der Phasen der Lehrerausbildung hinsichtlich der Vermittlung didaktischer und methodischer Kompetenzen für den schulischen Einsatz digitaler Medien in Deutschland und im Bundesländervergleich». In Schule digital - der Länderindikator 2016. Kompetenzen von Lehrpersonen der Sekundarstufe I im Umgang mit digitalen Medien im Bundesländervergleich, herausgegeben von Wilfried Bos, Ramona Lorenz, Manuela Endberg, Birgit Eickelmann, Rudolf Kammerl und Stefan Welling, 148-179. Münster: Waxmann. https://doi.org/10.25656/01:15134.

Fend, Helmut. 1980. Theorie der Schule. München, Wien, Baltimore: Urban \& Schwarzenberg.

Friedrichsen, Mike, und Wulf Wersig. 2020. «Digitale Kompetenz - Notwendigkeit und Kerngedanken». In Digitale Kompetenz. Herausforderungen für Wissenschaft, Wirtschaft, Gesellschaft und Politik. Synapsen im digitalen Informations- und Kommunikationsnetzwerk, herausgegeben von Mike Friedrichsen und Wulf Wersig, 3-6. Wiesbaden: Springer Gabler. https://doi.org/10.1007/978-3-658-22109-6_1.

Gerick, Julia, Mario Vennemann, Birgit Eickelmann, Wilfried Bos, und Sina Mews. 2018. ICILS 2013 - Dokumentation der Erhebungsinstrumente der International Computer and Information Literacy Study. Münster: Waxmann.

Getto, Barbara, und Kathrin Schulenburg. 2018. «Digitalisierung im Kontext strategischer Hochschulentwicklung an den Hochschulen in Nordrhein-Westfalen». In Digitalisierung und Hochschulentwicklung. Proceedings zur 26. Tagung der Gesellschaft für Medien in der Wissenschaft e.V., herausgegeben von Barbara Getto, Patrick Hintze und Michael Kerres, 36-48. Münster, New York: Waxmann. https://doi.org/10.25656/01:16998. 
Getto, Barbara, und Michael Kerres. 2015. «Vom E-Learning Projekt zur nachhaltigen Hochschulentwicklung: Strategisches Alignment im Kernprozess „Studium \& Lehre“»In Hochschulwege 2015. Wie verändern Projekte die Hochschulen? Herausgegeben von Andreas Mai, 147-157. Weimar: tredition. https://doi.org/10.25643/bauhaus-universitaet.3826.

Gidion, Gerd, und Michael Weyrich. 2017. «Mediale Hochschul-Perspektiven 2020 in Baden-Württemberg». Karlsruhe: KIT Scientific Publishing. https://doi.org/10.5445/ KSP/1000064688.

Gilch, Harald, Anna Sophie Beise, René Krempkow, Marko Müller, Friedrich Stratmann, und Klaus Wannemacher. 2019. «Digitalisierung der Hochschulen: Ergebnisse einer Schwerpunktstudie für die Expertenkommission Forschung und Innovation. Studien zum deutschen Innovationssystem Nr. 14-2019». Berlin: Expertenkommission Forschung und Innovation (EFI). http://hdl.handle.net/10419/194284.

Grosch, Michael. 2012. Mediennutzung im Studium. Eine empirische Untersuchung am Karlsruher Institut für Technologie. Aachen: Shaker.

Grosch, Michael und Gerd Gidion. 2011. Mediennutzungsgewohnheiten im Wandel. Karlsruhe: KIT Scientific Publishing. https://doi.org/10.5445/KSP/1000022524.

Goode, Johanna. 2010. «The Digital Identity Divide: How Technology Knowledge Impacts College Students». New Media Society 12, 497-513. https://doi.org/10.1177/1461444809343560.

Günther, Franziska. 2016. «Zur studentischen Nutzung von E-Learning- und Social Web-Anwendungen im universitären Kontext - eine explorative Analyse». Dresden: Technische Universität Dresden. https://nbn-resolving.org/urn:nbn:de:bsz:14-qucosa-235060.

Helmke, Andreas, und Franz E. Weinert. 1997. «Bedingungsfaktoren schulischer Leistungen». In Psychologie des Unterrichts und der Schule (Enzyklopädie der Psychologie, Pädagogische Psychologie; Vol.3.), herausgegeben von Franz E. Weinert, 71-176. Göttingen: Hogrefe.

Herzig, Bardo, und Alexander Martin. 2018. «Lehrerbildung in der digitalen Welt». In Digitalisierung und Bildung, herausgegeben von Silke Ladel, Julia Knopf und Armin Weinberger, 89-113. Wiesbaden: Springer VS. https://doi.org/10.1007/978-3-658-18333-2_6.

Hochschulforum Digitalisierung. 2016. «Zur nachhaltigen Implementierung von Lerninnovationen mit digitalen Medien». Berlin: Hochschulforum Digitalisierung. https://hochschulforumdigitalisierung.de/sites/default/files/dateien/HFD\%20AP\%20Nr\%2016_Grundlagentext\%20Change\%20Management.pdf.

Hofhues, Sandra, Sabrina Pensel, und Felix Möller. 2018. «Begrenzte Hochschulentwicklung. Das Beispiel digitaler Lerninfrastrukturen.» In Digitalisierung und Hochschulentwicklung. Proceedings zur 26. Tagung der Gesellschaft für Medien in der Wissenschaft e.V., herausgegeben von Barbara Getto, Patrick Hintze und Michael Kerres, 49-59. Münster, New York: Waxmann. https://doi.org/10.25656/01:16860.

Hofhues, Sandra, Mandy Schniefer-Rohs, Sandra Aßmann, und Taiga Brahm. 2020. «Studentische Medienwelten (in) der Gegenwart». In Studierende - Medien - Universität. Einblicke in die studentische Medienwelten, herausgegeben von Sandra Hofhues, Mandy SchnieferRohs, Sandra Aßmann und Taiga Brahm, 9-22. Münster, New York: Waxmann. https://doi. org/10.31244/9783830990499. 
Hugger, Kai-Uwe. 2008. «Medienkompetenz». In Handbuch Medienpädagogik, herausgegeben von Uwe Sander, Friederike Gross und Kai-Uwe Hugger, 93-99. Wiesbaden: Springer VS. https://doi.org/10.1007/978-3-531-91158-8_10.

Kammerl, Rudolf, und Kerstin Mayrberger. 2011. «Medienpädagogik in der Lehrerinnen- und Lehrerbildung in Deutschland: Aktuelle Situation und Desiderata». Beiträge zur Lehrerinnen- und Lehrerbildung 29 (2): 172-184. https://doi.org/10.25656/01:13776.

Kleimann, Bernd, und Klaus Wannemacher. 2004. «E-Learning an deutschen Hochschulen. Von der Projektentwicklung zur nachhaltigen Implementierung: Hochschulplanung Band 165». Hannover: HIS GmbH. https://his-he.de/fileadmin/user_upload/Publikationen/Projektberichte_alte_Website/Hochschulplanung/hp165.pdf.

KMK. 2017. «Bildung in der digitalen Welt. Strategie der Kultusministerkonferenz. Beschluss der Kultusministerkonferenz vom 08.12.2016 in der Fassung vom 07.12.2017». Berlin: Sekretariat der Kultusministerkonferenz. https://www.kmk.org/fileadmin/pdf/PresseUndAktuelles/2018/Digitalstrategie_2017_mit_Weiterbildung.pdf.

KMK. 2019. «Empfehlungen zur Digitalisierung in der Hochschullehre. Beschluss der Kultusministerkonferenz vom 14.03.2019». Berlin: Sekretariat der Kultusministerkonferenz. https:// www.kmk.org/fileadmin/Dateien/pdf/PresseUndAktuelles/2019/BS_190314_Empfehlungen_Digitalisierung_Hochschullehre.pdf.

Kommer, Sven, und Ralf Biermann. 2012. "Der mediale Habitus von (angehenden) Lehrerlnnen. Medienbezogene Dispositionen und Medienhandeln von Lehramtsstudierenden». In Jahrbuch Medienpädagogik 9, herausgegeben von Renate Schulz-Zander, Birgit Eickelmann, Heinz Moser, Horst Niesyto und Petra Grell, 81-109. Wiesbaden: VS Verlag für Sozialwissenschaften. https://doi.org/10.1007/978-3-531-94219-3_5.

Krauskopf, Karsten, und Carmen Zahn. 2015. «Differente Paradigmen digitalen Lernens als Grundlage für die Gestaltung akademischer Lehre». In Gestaltungsraum Hochschullehre. Potenziale nicht-traditionell Studierender nutzen, herausgegeben von Benjamin Klages, Marion Bonillo, Stefan Reinders und Axel Bohmeyer, 105-120. Opladen, Berlin, Toronto: Budrich UniPress. https://doi.org/10.25656/01:11437.

Özbek, Volkan, Ümit Alnıaçık, Faith Koc, M. Emin Akkılıçd und Eda Kaş. 2014. «The Impact of Personality on Technology Acceptance: A Study on Smart Phone Users». Procedia - Social and Behavioral Sciences 150, 541-551. https://doi.org/10.1016/j.sbspro.2014.09.073.

Palkowitsch-Kühl, Jens. 2018. «Digitale Medienkompetenz - eine Schlüsselkompetenz in der Lehrkräfteausbildung. Aktuelle Perspektiven von Lehrkräften im Bereich schulisch verantworteter religiöser Bildung». Zeitschrift für Pädagogik und Theologie 70 (3), 294-307. https://doi.org/10.1515/zpt-2018-0035.

Pensel, Sabrina, und Sandra Hofhues. 2017. «Digitale Lerninfrastrukturen an Hochschulen. Systematisches Review zu den Rahmenbedingungen für das Lehren und Lernen mit Medien an deutschen Hochschulen». http://your-study.info/wp-content/uploads/2018/01/ Review_Pensel_Hofhues.pdf. 
Persike, Malte, und Julius-David Friedrich. 2016. «Lernen mit digitalen Medien aus Studierendenperspektive. Arbeitspapier Nr. 17». Berlin: Hochschulforum Digitalisierung. https:// hochschulforumdigitalisierung.de/sites/default/files/dateien/HFD_AP_Nr_17_Lernen_ mit_digitalen_Medien_aus_Studierendenperspektive.pdf.

Petko, Dominik, und Beat Döbeli Honegger. 2011. «Digitale Medien in der schweizerischen Lehrerinnen- und Lehrerbildung: Hintergründe, Ansätze und Perspektiven». Beiträge zur Lehrerinnen- und Lehrerbildung 29 (2), 155-171. https://doi.org/10.25656/01:13775.

Pumptow, Marina, und Taiga Brahm. 2020. «Erkenntnisse zur medienbezogenen Selbstwirksamkeit von Studierenden». In Studierende - Medien - Universität. Einblicke in die studentische Medienwelten, herausgegeben von Sandra Hofhues, Mandy Schniefer-Rohs, Sandra Aßmann und Taiga Brahm, 107-130. Münster, New York: Waxmann. https://doi. org/10.31244/9783830990499.

Riedel, Jana, und Claudia Börner. 2018. «Wir tun es, weil es gut ist! Wie Lehrende die Erfolgsfaktoren für den Einsatz digitaler Medien in der Hochschullehre einschätzen». In Teaching Trends 2016. Digitalisierung in der Hochschule: Mehr Vielfalt in der Lehre, Wolfgang Pfau, Caroline Baetge, Svenja Mareike Bedenlier, Carina Kramer und Joachim Stöter, 209-220. Münster: Waxmann. https://doi.org/10.25656/01:18900.

Riplinger, Tim, und Mandy Schiefner-Rohs. 2017. Medieneinsatz in der Hochschullehre. Akademische Lehr-Lernkonzepte zwischen Zumutung und Zu-Mutung. Köln: Universität zu Köln, Humanwissenschaftliche Fakultät, Department Erziehungs- und Sozialwissenschaften. https://doi.org/10.13154/rub.105.94.

Rott, Karin Julia. 2014. «Medienkompetenz im Studium: Wie gut ist die Vorbereitung für das spätere Berufsfeld?»In Teaching Trends 2014. Offen für neue Wege: Digitale Medien in der Hochschule, herausgegeben von Olaf Zawacki-Richter, David Kergel, Norbert Kleinefeld, Petra Muckel, Joachim Stöter und Katrin Brinkmann, 153-169. Münster: Waxmann. https:// doi.org/10.25656/01:18467.

Schäffer, Dennis. 2015. «E-Learning als Teil des persönlichen, intentionalen Lernraumes im Studium. Eine explorative Studie an Studierenden an der Fakultät für Erziehungswissenschaft an der Universität Bielefeld». Berlin: Epubli GmbH. https://doi.org/10.25656/01:10912.

Schmid, Ulrich, und Berit Bäßler. 2016. «Strategieoptionen für Hochschulen im digitalen Zeitalter. Arbeitspapier Nr. 29». Berlin: Hochschulforum Digitalisierung. https://hochschulforumdigitalisierung.de/sites/default/files/dateien/HFD_AP_Nr29_Strategieoptionen_ fuer_Hochschulen_im_digitalen_Zeitalter.pdf.

Schmid, Ulrich, Lutz Goertz, Sabine Radomski, Sabrina Thom, und Julia Behrens. 2017. «Monitor Digitale Bildung. Die Hochschulen im digitalen Zeitalter». Gütersloh. https://www. bertelsmann-stiftung.de/fileadmin/files/BSt/Publikationen/GrauePublikationen/DigiMonitor_Hochschulen_final.pdf.

Steffens, Yannic, Inga Lotta Schmitt, und Sandra Aßmann. 2017. «Mediennutzung Studierender: Über den Umgang mit Medien in hochschulischen Kontexten. Systematisches Review nationaler und internationaler Studien zur Mediennutzung Studierender». Köln: Universität zu Köln, Humanwissenschaftliche Fakultät, Department Erziehungs- und Sozialwissenschaften. https://doi.org/10.13154/rub.106.95. 
Süss, Daniel, Claudia Lampert, und Christine Trueltzsch-Wijnen. 2018. Medienpädagogik. Ein Studienbuch zur Einführung, (3. Auflage). Wiesbaden: Springer VS. https://doi. org/10.1007/978-3-658-19824-4.

Taylor, Shirley, und Peter A. Todd. 1995. «Understanding information technology usage: A test of competing models». Information Systems Research 2 (6), 144-176. https://doi. org/10.1287/isre.6.2.144.

Vogelsang, Christoph, Alexander Finger, Daniel Laumann, und Christoph Thyssen. 2019. «Vorerfahrungen, Einstellungen und motivationale Orientierungen als mögliche Einflussfaktoren auf den Einsatz digitaler Werkzeuge im naturwissenschaftlichen Unterricht». ZfDN 25, 115-129. https://doi.org/10.1007/s40573-019-00095-6.

Von der Heyde, Markus, Gunnar Auth, Andreas Hartmann, und Christian Erfurth. 2017. «Hochschulentwicklung im Kontext der Digitalisierung - Bestandsaufnahme, Perspektiven, Thesen». In INFORMATIK 2017, herausgegeben von Maximilian Eibl und Martin Gaedke, 17571772. Bonn: Gesellschaft für Informatik. https://doi.org/10.18420/in2017_175.

Wannemacher, Klaus, Imke Jungermann, Julia Scholz, Hacer Tercanli, und Anna von Villiez. 2016. «Digitale Lernszenarien im Hochschulbereich. Arbeitspapier Nr. 15». Berlin: Hochschulforum Digitalisierung. https://hochschulforumdigitalisierung.de/sites/default/files/ dateien/HFD\%20AP\%20Nr\%2015_Digitale\%20Lernszenarien.pdf.

Wedekind, Joachim 2008. «Medienkompetenz für (Hochschul-)Lehrende». Zeitschrift für ELearning 3 (2), 24-37.

Werner, Benita. 2006. «Status des E-Learning an deutschen Hochschulen». https://www.eteaching.org/projekt/fallstudien/Status_des_ELearning.pdf.

Wissenschaftsrat. 2017. «Strategien für die Hochschullehre. Positionspapier». Halle (Saale): Wissenschaftsrat. https://www.wissenschaftsrat.de/download/archiv/6190-17.pdf.

Wolf, Thomas und Jaqueline-Helena Strohchen. 2018. «Digitalisierung: Definition und Reife». Informatik Spektrum 41 (1), 56-64. https://doi.org/10.1007/s00287-017-1084-8.

Zawacki-Richter, Olaf, Carina Cramer, und Wolfgang Müskens. 2016. «Studiumsbezogene Mediennutzung im Wandel - Querschnittdaten 2012 und 2015 im Vergleich». Schriftenreihe zum Bildungs- und Wissenschaftsmanagement, 01/2016, Oldenburg. https://openjournal. uni-oldenburg.de/index.php/bildungsmanagement/article/view/101. 